\title{
Quantitative mechanisms of DNA damage sensing and signaling
}

\author{
Susanne C. S. Bantele ${ }^{1}\left[\right.$ ] Boris Pfander ${ }^{1}(\mathbb{C}$
}

Received: 29 May 2019 / Revised: 13 June 2019 / Accepted: 14 June 2019 / Published online: 21 June 2019

(C) The Author(s) 2019

\begin{abstract}
DNA damage occurs abundantly during normal cellular proliferation. This necessitates that cellular DNA damage response and checkpoint pathways monitor the cellular DNA damage load and that DNA damage signaling is quantitative. Yet, how DNA lesions are counted and converted into a quantitative response remains poorly understood. We have recently obtained insights into this question investigating DNA damage signaling elicited by single-stranded DNA (ssDNA). Intriguingly, our findings suggest that local and global DNA damage signaling react differentially to increasing amounts of DNA damage. In this mini-review, we will discuss these findings and put them into perspective of current knowledge on the DNA damage response.
\end{abstract}

Keywords DNA damage checkpoint · Signal transduction · Double strand break $\cdot$ DNA end resection $\cdot$ Cell cycle $\cdot$ Posttranslational modification $\cdot$ Genome stability

In order to ensure genome stability, cells need to detect DNA lesions such as DNA double-stranded breaks (DSBs) and signal their presence so that an appropriate cellular response is triggered (Zhou and Elledge 2000; Harrison and Haber 2006; Ciccia and Elledge 2010). The central importance of the networks carrying out this fundamental task is underscored by the observation that mutations in DNA damage signaling proteins often coincide with a predisposition for cancer development and progeria (O'Driscoll 2012).

DNA damage signaling is commonly understood to be a quantitative process, which generates a response appropriate to the cellular damage load (Zierhut and Diffley 2008; Balogun et al. 2013; Clerici et al. 2014; Ira et al. 2004; Mantiero et al. 2007). It is, however, unclear how such a quantitative response is generated in molecular terms and how the dynamic range of the response is tuned.

DNA damage signaling is mediated by proteins of the DNA damage checkpoint, which can recognize DNA structures that indicate the presence of DNA lesions and convert them into downstream DNA damage signals. Single-stranded

Communicated by M. Kupiec.

Boris Pfander

bpfander@biochem.mpg.de

1 Max Planck Institute of Biochemistry, DNA Replication and Genome Integrity, Martinsried, Germany
DNA (ssDNA) marks sites of DNA damage and can be considered an upstream DNA damage signal that is recognized by proteins of the DNA damage checkpoint in order to transduce this upstream signal into a downstream checkpoint signal. While ssDNA is inherently generated during DNA replication and transcription, the presence of DNA lesions often triggers extensive ssDNA formation (Zou 2007). For example, at DSBs ssDNA is generated by a process called DNA end resection (Sugawara and Haber 1992; Symington 2014).

What information does the amount of ssDNA in a cell hold? First, it gives information about the number of lesion sites, as many lesions will obviously expose more ssDNA than few. Second, it could potentially tell us about the persistence time of a given DNA lesion, at least in the case of DSBs (Pellicioli et al. 2001). The longer a lesion remains unrepaired, the more time for processing and production of ssDNA. Long persistence can perhaps be taken as indication of lesions difficult to repair. Third, ssDNA is generated in S phase upon stalling or nucleolytic processing of replication forks (Lopes et al. 2006; Sogo et al. 2002). All three scenarios-a high number of DNA lesions, the presence of persistent lesions and the occurence of DNA lesions or stalled/ broken replication forks in S phase-can be seen as severe threat to cellular survival calling for a cell-wide response and activation of the DNA damage checkpoint. It therefore seems plausible that cells possess a counting mechanism for 
the ssDNA signal and that the overall amount of ssDNA in a given cell must overcome a threshold to activate the DNA damage checkpoint.

How is the ssDNA signal read and translated into a downstream DNA damage checkpoint signal? Mechanistically, ssDNA in cells is bound by RPA (Wold 1997; Chen and Wold 2014), which in budding yeast directly interacts with the DNA damage kinase Mec1 (ATR in humans) and its co-factor Ddc2 (ATRIP in humans) (Zou and Elledge 2003; Cortez et al. 2001; Paciotti et al. 2000). More ssDNA will attract more Mec1-Ddc2 (Zou and Elledge 2003; Nakada et al. 2004; Bantele et al. 2019) suggesting an intuitive mechanism of how ssDNA could be quantified. Mec1-Ddc2 phosphorylates different target proteins. Interestingly, however, not all proteins targeted by Mec1-Ddc2 show the same dependency on the ssDNA signal. In our recent work, we define two Mec1 signaling circuits, which respond differently to quantitatively different amounts of ssDNA (Bantele et al. 2019). One circuit activates the DNA damage effector kinase Rad53, which mediated by its co-sensors and scaffolds is recruited and activated in a manner that strongly depends on the ssDNA length and therefore integrates over the ssDNA signal (Bantele et al. 2019; Ira et al. 2004). As Rad53 sets off the cell-wide DNA damage checkpoint (de Oliveira et al. 2015; Harrison and Haber 2006; Branzei and Foiani 2006), we call this response the "global" signaling circuit (Fig. 1a, left). A second circuit leads to phosphorylation of the histone $\mathrm{H} 2 \mathrm{~A}(\gamma \mathrm{H} 2 \mathrm{~A})$, which forms a chromatin domain surrounding the DNA lesion (Shroff et al. 2004; Rogakou et al. 1998) and behaves fundamentally different. $\gamma \mathrm{H} 2 \mathrm{~A}$ phosphorylation appears to be full-blown even at very low amounts of ssDNA signal and is seemingly unresponsive to further ssDNA accumulation (Bantele et al. 2019). Notably, $\gamma \mathrm{H} 2 \mathrm{~A}$ is not involved in the cell-wide response but rather thought to facilitate local changes in the damaged chromosome and perhaps promote repair (Downs et al. 2004; Kim et al. 2007; Kruhlak et al. 2006; Redon et al. 2003; Tsabar et al. 2015). We therefore termed this response the "local" signaling circuit (Fig. 1a, right). As both circuits are triggered by the same kinase-Mec1-Ddc2, a model emerges by which different Mec1 kinase targets have different sensitivities towards the kinase. While $\gamma \mathrm{H} 2 \mathrm{~A}$, in the center of the local response, is efficiently targeted already at very low levels of damage-associated Mec1 kinase, activation of the DNA damage checkpoint effector kinase Rad53, which acts globally, requires accumulation of ssDNA-RPA (Bantele et al. 2019). Such a separation of responses at different amounts of the ssDNA signal seems to be a reliable strategy to ensure immediate full-blown local repair while launching a cost-intense, cell-wide checkpoint response including cell cycle arrest only if the damage load is high or DNA lesions are persistent and a safe passage through the cell cycle is no longer guaranteed.
A

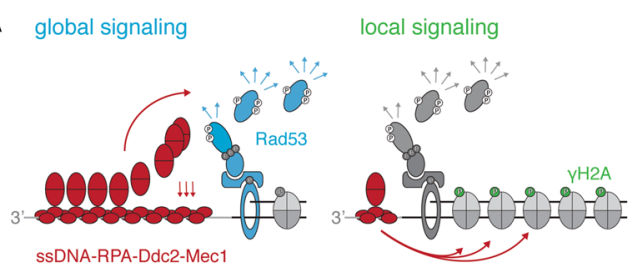

B

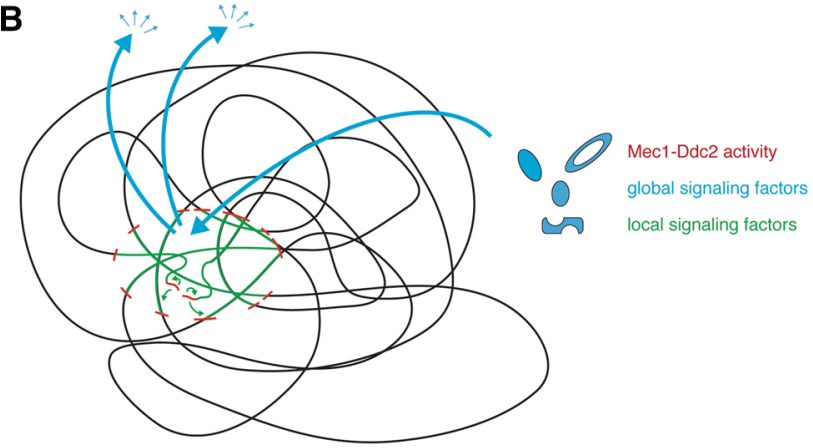

Fig. 1 Two distinct signaling circuits operate DNA damage signaling. a Schematic representation of the two Mec1-Ddc2-dependent signaling circuits within the DNA damage checkpoint. The global signaling circuit (left) integrates the quantitative information of the initial ssDNA-RPA signal and over a wide range of signal translates it into a proportional amount of activated Rad53 effector kinase. The local signaling circuit (right) is already fully active at very low amounts of ssDNA-RPA signal and triggers the local response. b Putative model of the different mechanisms by which substrates of the local (green) and global (blue) checkpoint signaling circuits access Mec1 activity (red), which in case of local signaling is likely determined by architectural characteristics of the chromatin

The concept of signaling thresholds separating different cellular responses can be observed already in the bacterial SOS response (Michel 2005; Maslowska et al. 2019). The SOS response also monitors ssDNA accumulation and ssDNA-RecA binding and signals it via the LexA transcriptional repressor system. Here, the LexA-dependent repression of repair genes is gradually removed upon increasing RecA-ssDNA accumulation/DNA damage. At low doses of ssDNA, genes involved in nucleotide excision repair (NER) are de-repressed (Courcelle et al. 2001; Michel 2005). Further ssDNA accumulation then leads to de-repression of TLS genes and an inhibitor of cell division, which can be seen as a mechanism to stop cellular proliferation analogous to the checkpoint in eukaryotes (Tippin et al. 2004). In this case, the transition from proliferation to arrest is therefore accompanied by an additional transition from NER to mutagenic TLS (Michel 2005; Tippin et al. 2004; Courcelle et al. 2001). Interestingly, quantitative read-out of the ssDNA signal therefore seems to be a universal feature of prokaryotic and eukaryotic DNA damage responses suggesting that a decision whether DNA damage levels are tolerable or a 
full-blown cell-wide DNA damage response is required in all cells.

The differentiation of signaling circuits brings up several interesting questions regarding the underlying molecular mechanisms. How is Mec1 kinase activity differentially channeled towards different Mec1 targets? How is the $\gamma \mathrm{H} 2 \mathrm{~A}$ domain established efficiently with low levels of DNA damage-associated kinase? How are thresholds that are locally defined at every lesion site integrated over spatially distinct lesion sites?

One fundamental difference between targets in the local and global circuits is their availability to phosphorylation by the Mec1 kinase. The global signaling cascade constituted by the 9-1-1 co-sensor, the Dpb11 and Rad9 scaffold proteins and the Rad53 effector kinase requires several steps of protein recruitment to the DNA lesion site, which itself is dependent on DNA end resection and Mec1 (Ira et al. 2004; Ma et al. 2006; Puddu et al. 2008). It therefore seems reasonable to suggest that protein recruitment requirements dictate subsequent phosphorylation by Mec1. In case of $\gamma \mathrm{H} 2 \mathrm{~A}$, the scenario is fundamentally different. $\mathrm{H} 2 \mathrm{~A}$ is an integral component of chromatin and as such immediately available as substrate for phosphorylation. Therefore, and despite the fact that histones were shown to display plasticity at DSBs (Hauer et al. 2017; Hauer and Gasser 2017; Adam et al. 2016; Tsabar et al. 2016), encounters between Mec1 and H2A over the broad $\gamma \mathrm{H} 2 \mathrm{~A}$ domain will rather be defined by chromatin architecture and mobility than recruitment (Caron et al. 2015; Aymard and Legube 2016; Lee et al. 2013; Renkawitz et al. 2013; Zimmer and Fabre 2019). Taken together, we therefore propose that Mec1 targets are phosphorylated dependent on the frequency of kinase-substrate encounters, which in the global signaling circuit is determined by recruitment and in the local signaling circuit depends on chromatin architecture (Fig. 1b).

Based on this model, the Mec1-Ddc2 sensor module is critically involved in all ssDNA signaling circuits and likely involved in transducing the quantitative nature of the ssDNA signal. However, a second sensing mechanism might be required to confer the amount of ssDNA in the global signaling circuit. Currently, the best candidates for this second sensor are the 9-1-1 clamp and factors associated with it (Bantele et al. 2019). It has been shown that 9-1-1 is recruited to the border of resection (Majka et al. 2006; Majka and Burgers 2007), and we could observe resectiondependent 9-1-1 accumulation. However, by which molecular mechanism the 9-1-1 complex or its associated factors are involved in sensing or transducing the ssDNA signal will need to be determined by future research.

We therefore suggest that the cellular DNA damage response should not be viewed as a single pathway, but rather as separable signaling circuits. Different DNA damage scenarios trigger differential responses in these individual circuits. A quantitative understanding of the signal transduction in the individual circuits is therefore required to understand and manipulate cellular decision making upon DNA damage.

Acknowledgements Open access funding provided by Max Planck Society.

Funding The funding was received by Max-Planck-Gesellschaft (DE), Deutsche Forschungsgemeinschaft (DE) (Grant no. PF794-1/1).

Open Access This article is distributed under the terms of the Creative Commons Attribution 4.0 International License (http://creativeco mmons.org/licenses/by/4.0/), which permits unrestricted use, distribution, and reproduction in any medium, provided you give appropriate credit to the original author(s) and the source, provide a link to the Creative Commons license, and indicate if changes were made.

\section{References}

Adam S et al (2016) Real-time tracking of parental histones reveals their contribution to chromatin integrity following DNA damage. Mol Cell 64(1):1-15

Aymard F, Legube G (2016) A TAD closer to ATM. Mol Cell Oncol $3(3): 1-3$

Balogun FO, Truman AW, Kron SJ (2013) DNA resection proteins Sgs1 and Exo1 are required for G1 checkpoint activation in budding yeast. DNA Repair 12(9):1-10

Bantele SCS, Lisby M, Pfander B (2019) Quantitative sensing and signalling of single-stranded DNA during the DNA damage response. Nat Commun 10(1):944

Branzei D, Foiani M (2006) The Rad53 signal transduction pathway: replication fork stabilization, DNA repair, and adaptation. Exp Cell Res 312(14):2654-2659

Caron P et al (2015) Non-redundant functions of ATM and DNAPKcs in response to DNA double-strand breaks. Cell Rep 13(8):1598-1609

Chen R, Wold MS (2014) Replication protein A: single-stranded DNA's first responder. BioEssays 36(12):1156-1161

Ciccia A, Elledge SJ (2010) The DNA damage response: making it safe to play with knives. Mol Cell 40(2):179-204

Clerici M, Trovesi C, Galbiati A, Lucchini G, Longhese MP (2014) Mec1/ATR regulates the generation of single-stranded DNA that attenuates Tel1/ATM signaling at DNA ends. EMBO J 33(3): 198-216

Cortez D et al (2001) ATR and ATRIP: partners in checkpoint signaling. Science 294(5547):1713-1716

Courcelle J et al (2001) Comparative gene expression profiles following UV exposure in wild-type and SOS-deficient Escherichia coli. Genetics 158(1):41-64

de Oliveira FMB et al (2015) Phosphoproteomics reveals distinct modes of Mec1/ATR signaling during DNA replication. Mol Cell 57(6):1-10

Downs JA et al (2004) Binding of chromatin-modifying activities to phosphorylated histone $\mathrm{H} 2 \mathrm{~A}$ at DNA damage sites. Mol Cell 2004(16):979-990

Harrison JC, Haber JE (2006) Surviving the breakup: the DNA damage checkpoint. Annu Rev Genet 40(1):209-235

Hauer MH, Gasser SM (2017) Chromatin and nucleosome dynamics in DNA damage and repair. Genes Dev 31(22):2204-2221 
Hauer MH et al (2017) Histone degradation in response to DNA damage enhances chromatin dynamics and recombination rates. Nat Struct Mol Biol 24(2):99-107

Ira $\mathrm{G}$ et al (2004) DNA end resection, homologous recombination and DNA damage checkpoint activation require CDK1. Nature 431(7011):1011-1017

Kim J-A et al (2007) Heterochromatin is refractory to gamma-H2AX modification in yeast and mammals. J Cell Biol 178(2):209-218

Kruhlak MJ et al (2006) Changes in chromatin structure and mobility in living cells at sites of DNA double-strand breaks. J Cell Biol 172(6):823-834

Lee CS et al (2013) Dynamics of yeast histone H2A and H2B phosphorylation in response to a double-strand break. Nat Strcut Mol Biol 21(1):103-109. https://doi.org/10.1038/nsmb.2737

Lopes M, Foiani M, Sogo JM (2006) Multiple mechanisms control chromosome integrity after replication fork uncoupling and restart at irreparable UV lesions. Mol Cell 21(1):15-27

Ma J-L et al (2006) Activation of the checkpoint kinase Rad53 by the phosphatidyl inositol kinase-like kinase Mec1. J Biol Chem 281(7):3954-3963

Majka J, Burgers PMJ (2007) Clamping the Mec1/ATR checkpoint kinase into action. Cell Cycle (Georgetown, Tex.) 6(10):1157-1160

Majka J et al (2006) Replication protein a directs loading of the DNA damage checkpoint clamp to 5'-DNA junctions. J Biol Chem 281(38):27855-27861

Mantiero D et al (2007) Dual role for Saccharomyces cerevisiae Tel1 in the checkpoint response to double-strand breaks. EMBO Rep 8(4):380-387

Maslowska KH, Makiela Dzbenska K, Fijalkowska IJ (2019) The SOS system: a complex and tightly regulated response to DNA damage. Environ Mol Mutagen 60(4):368-384

Michel B (2005) After 30 years of study, the bacterial SOS response still surprises us. PLoS Biol 3:e255

Nakada D, Hirano Y, Sugimoto K (2004) Requirement of the Mre11 complex and exonuclease 1 for activation of the Mec1 signaling pathway. Mol Cell Biol 24(22):10016-10025

O'Driscoll M (2012) Diseases associated with defective responses to DNA damage. Cold Spring Harb Perspect Biol 4(12):a012773-a012773

Paciotti V et al (2000) The checkpoint protein Ddc2, functionally related to $S$. pombe Rad26, interacts with Mec1 and is regulated by Mec1-dependent phosphorylation in budding yeast. Genes Dev 14(16):2046-2059

Pellicioli A et al (2001) Regulation of Saccharomyces Rad53 checkpoint kinase during adaptation from DNA damage-induced G2/M arrest. Mol Cell 7(2):293-300

Puddu F et al (2008) Phosphorylation of the budding yeast 9-1-1 complex is required for Dpb11 function in the full activation of the UV-induced DNA damage checkpoint. Mol Cell Biol 28(15):4782-4793. https://doi.org/10.1128/MCB.00330-08
Redon C et al (2003) Yeast histone 2A serine 129 is essential for the efficient repair of checkpoint-blind DNA damage. EMBO Rep 4(7):678-684

Renkawitz J, Lademann CA, Jentsch S (2013) $\gamma \mathrm{H} 2 \mathrm{AX}$ spreading linked to homology search. Cell Cycle (Georgetown, Tex.) 12(16):2526-2527

Rogakou EP et al (1998) DNA double-stranded breaks induce histone $\mathrm{H} 2 \mathrm{AX}$ phosphorylation on serine 139. J Biol Chem 273(10):5858-5868

Shroff $\mathrm{R}$ et al (2004) Distribution and dynamics of chromatin modification induced by a defined DNA double-strand break. Curr Biol 14(19):1703-1711

Sogo JM, Lopes M, Foiani M (2002) Fork reversal and ssDNA accumulation at stalled replication forks owing to checkpoint defects. Science (New York, N.Y.) 297(5581):599-602. https://doi. org/10.1016/j.cub.2004.09.04

Sugawara N, Haber JE (1992) Characterization of double-strand break-induced recombination: homology requirements and singlestranded DNA formation. Mol Cell Biol 12(2):563-575

Symington LS (2014) End resection at double-strand breaks: mechanism and regulation. Cold Spring Harb Perspect Biol 6(8):a016436

Tippin B, Pham P, Goodman MF (2004) Error-prone replication for better or worse. Trends Microbiol 12(6):288-295

Tsabar M et al (2015) Caffeine impairs resection during DNA break repair by reducing the levels of nucleases Sae2 and Dna2. Nucleic Acids Res 43(14):6889-6901

Tsabar M, Hicks WM, Tsaponina O, Haber JE (2016) Re-establishment of nucleosome occupancy during double-strand break repair in budding yeast. DNA Repair 47:21-29

Wold MS (1997) Replication protein A: a heterotrimeric, singlestranded DNA-binding protein required for eukaryotic DNA metabolism. Annu Rev Biochem 66(1):61-92

Zhou B, Elledge SJ (2000) The DNA damage response: putting checkpoints in perspective. Nature 408(6811):433-439

Zierhut C, Diffley JFX (2008) Break dosage, cell cycle stage and DNA replication influence DNA double strand break response. EMBO J 27(13):1875-1885

Zimmer C, Fabre E (2019) Chromatin mobility upon DNA damage: state of the art and remaining questions. Curr Genet 65(1):1-9

Zou L (2007) Single- and double-stranded DNA: building a trigger of ATR-mediated DNA damage response. Genes Dev 21(8):879-885

Zou L, Elledge SJ (2003) Sensing DNA damage through ATRIP recognition of RPA-ssDNA complexes. Science (New York, N.Y.) 300(5625):1542-1548

Publisher's Note Springer Nature remains neutral with regard to jurisdictional claims in published maps and institutional affiliations. 